\title{
On the Melan equation for suspension bridges
}

\author{
Filippo Gazzola, Mohamed Jleli and Bessem Samet \\ Filippo Gazzola \\ Dipartimento di Matematica \\ Politecnico di Milano \\ Piazza Leonardo da Vinci 32 \\ 20133 Milano \\ Italy \\ e-mail: filippo.gazzola@polimi.it \\ Mohamed Jleli \\ Department of Mathematics \\ King Saud University \\ Riyadh \\ Saudi Arabia \\ e-mail: jleli@ksu.edu.sa \\ Bessem Samet \\ Department of Mathematics \\ King Saud University \\ Riyadh \\ Saudi Arabia \\ e-mail: bsamet@ksu.edu.sa
}

\section{Introduction and historical overview}

The celebrated report by Navier [18], published in 1823, was for several decades the only mathematical treatise of suspension bridges. It mainly deals with the static of cables and their interaction with towers: some second-order ordinary differential equations are derived and solved. At that time, no stiffening trusses had yet appeared and the models suggested by Navier are oversimplified in several aspects. In spite of a lack of prior history, the report by Navier appears as a masterpiece of amazing precision, including a part of applications intended to suggest how to plan some suspension bridges; see [18, Troisième Partie].

In the 19th century some further contributions deserve to be mentioned. The theory of structures, contained in the monograph by Rankine [23], makes an analysis of the general principles governing chains, cords, ribs and arches; 
the part on suspension bridge with sloping rods [23, pp. 171-173] makes questionable assumptions and rough approximations. As far as we are aware, this contribution has not been applied to real bridges. In 1875, Castigliano [4] suggested a new theory for elastic systems close to equilibrium and proved a result known nowadays as the Castigliano theorem; this theorem became the core of his main work [5] published in 1879. His method allows to study the deflection of structures by strain energy method. His theorem of the derivatives of internal work of deformation extended its application to the calculation of relative rotations and displacements between points in the structure and to the study of beams in flexure.

A milestone theoretical contribution to suspension bridges is the monograph by the Austrian engineer Melan [17], whose first edition goes back to 1888. This book was translated to English by Steinman who, in the preface to his translation, wrote "The work has been enthusiastically received in Europe where it has already gone through three editions and the highest honors have been awarded the author." Melan considered the bridges with all those forms of construction having the characteristic of transmitting oblique forces to the abutments even when the applied loads are vertical in direction. Melan made a detailed study of the static of cables and beams through a careful analysis of the different kinds of suspension bridges according to the number of spans, the stiffened or unstiffened structure, the effect of temperature. He repeatedly used the Castigliano theorem, in particular for the computation of deflection [17, p. 69]. Melan [17, p. 77] suggested a fourth-order equation to describe the behavior of suspension bridges; he viewed a suspension bridge as an elastic beam suspended to a sustaining cable (see Figure 1 below) and his equation reads

$$
E I w^{\prime \prime \prime \prime}(x)-(H+h(w)) w^{\prime \prime}(x)+\frac{q}{H} h(w)=p(x) \quad \forall x \in(0, L),
$$

and it is the object of the present paper. In Section 2 we derive (1.1) in full detail and we explain the physical meaning of all the terms. von Kármán and Biot $[14,(5.5)]$ called (1.1) the fundamental equation of the theory of the suspension bridge.

It is our purpose to discuss the Melan equation (1.1) from several points of view. First of all, the term $h(w)$ (representing the additional tension of the sustaining cable due to live loads) makes (1.1) a nonlinear nonlocal equation and, for this reason, it is often considered as a constant in the engineering literature. However, the nonlinear structural behavior of suspension bridges is by now well established; see, e.g., [3, 10, 12, 15, 21]. Therefore, the term $h(w)$ deserves a special attention. In Section 3 we give a survey of the possible forms of $h$ usually considered in literature, while in Section 4 we discuss the differences between these forms; it turns out that there may be significant discrepancies.

In Section 5 we prove existence results for (1.1) by applying some fixed point theorems. A fairly wide class of nonlocal terms $h(w)$ is considered. Since 


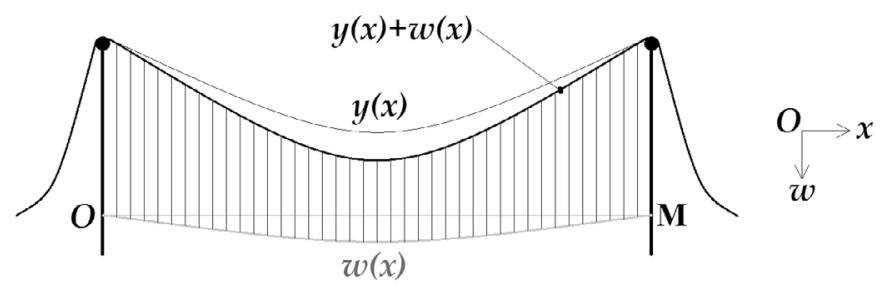

FiguRE 1. Beam (grey) sustained by a cable (black) through parallel hangers.

we were unable to prove general uniqueness results, we sought a counterexample: we found a particular equation (1.1) admitting two solutions, a small one and a larger one. This raises some doubts about well-posedness of (1.1).

The Melan equation (1.1) has also attracted the interest of numerical analysts; see [8, 20, 24, 25, 30]. In these papers, several approximating procedures for the solution of (1.1) have been discussed for different forms of the term $h(w)$. In view of the above-mentioned counterexample to uniqueness, one expects iterative numerical procedures to be quite unstable. In Section 6 we suggest a unifying approach for equation (1.1) for a wide class of nonlocal terms $h(w)$. We set up a fixed point iterative method which enables us to control the convergence of the approximating terms $h\left(w_{n}\right)$, where $\left\{w_{n}\right\}$ is a sequence of possible approximations of the solution of (1.1). Some numerical results testify that our approach may be used to get good approximate solutions, provided the parameters lie in some suitable range. In Section 7 we numerically study (1.1) with parameters taken from an actual bridge, as suggested by Wollmann [30]: in this situation, fixed points appear to be quite unstable and a different iterative procedure is used.

This paper is organized as follows. In Section 2 we derive the classical Melan equation. In Section 3 we discuss three different approximations of the nonlocal term $h(w)$ suggested in literature. In Section 4 we compute the response of these approximations for some special forms of the beam. In Section 5 we state our existence results for the Melan equation (1.1), as well as a counterexample to uniqueness. In Sections 6 and 7 we give some numerical results relative to our approximation scheme. Sections 8-10 are devoted to the proofs of the existence results. Finally, Section 11 contains our conclusions and some open problems.

\section{The derivation of the Melan equation}

The classical deflection theory of suspension bridges models the bridge structure as a combination of a string (the sustaining cable) and a beam (the roadway); see Figure 1. We follow here [14, Section VII.1]. The point $O$ is the origin of the orthogonal coordinate system and positive displacements 
are oriented downwards. The point $M$ has coordinates $M(0, L)$, where $L$ is the distance between the two towers. When the system is only subject to the action of dead loads, the cable is in position $y(x)$, while the unloaded beam is the segment connecting $O$ and $M$. The cable is adjusted in such a way that it carries its own weight, the weight of the hangers and the dead weight of the roadway (beam) without producing a bending moment in the beam so that all additional deformations of the cable and the beam due to live loads are small. The cable is modeled as a perfectly flexible string subject to vertical dead and live loads. When the string is subject to a downwards vertical dead load $q(x)$, the horizontal component $H>0$ of the tension remains constant. If the mass of the cable (dead load) is neglected, then the load is distributed per horizontal unit. If we assume that spacing between hangers is small relative to the span, then the hangers can be considered as a continuous sheet or a membrane uniformly connecting the cable and the beam (live load). This is a simplified sketch of what occurs in a suspension bridge, provided that the mass of the cable is neglected and that the roadway is sought as a beam. The resulting equation reads (see $[14,(1.3)$, Section VII]):

$$
H y^{\prime \prime}(x)=-q(x) \text {. }
$$

If the endpoints of the string are at the same level $\gamma$ (as in suspension bridges, see Figure 1) and if the dead load is constant, $q(x) \equiv q$, then the solution of (2.1) and the length $L_{c}$ of the cable are given by

$$
\begin{aligned}
y(x) & =\gamma+\frac{q}{2 H} x(L-x), \\
L_{c} & =\int_{0}^{L} \sqrt{1+y^{\prime}(x)^{2}} d x \\
& =\frac{L}{2} \sqrt{1+\frac{q^{2} L^{2}}{4 H^{2}}}+\frac{H}{q} \log \left(\frac{q L}{2 H}+\sqrt{1+\frac{q^{2} L^{2}}{4 H^{2}}}\right) .
\end{aligned}
$$

Hence, the cable takes the shape of a parabola ( $y$ is positive downwards so that it has a $\cup$-shaped graph).

Summarizing, we denote by

- $L$ the length of the beam at rest (the distance between towers) and $x \in(0, L)$ the position on the beam;

- $q$ and $p=p(x)$ the dead and live loads per unit length applied to the beam;

- $y=y(x)$ the downwards displacement of the cable connecting the endpoints (at level $\gamma$ ), due to the dead load $q$;

- $L_{c}$ the length of the cable subject to the dead load $q$;

- $w=w(x)$ the downwards displacement of the beam and, hence, the additional displacement of the cable due to the live load $p$;

- $H$ the horizontal tension in the cable, when subject to the dead load $q$ only;

- $h=h(w)$ the additional tension in the cable produced by the live load $p$. 
The function $w$ describes both the downwards displacements of the beam and the cable because the elastic deformation of the hangers is neglected. This classical assumption is justified by precise studies on linearized models; see [16]. Since the dead load $q$ of the beam is constant, (2.2) yields

$$
y^{\prime \prime}(x)=-\frac{q}{H}, \quad y^{\prime}(x)=\frac{q}{H}\left(\frac{L}{2}-x\right) \quad \forall x \in(0, L) .
$$

When the live load $p$ is added, a certain amount $p_{1}$ of $p$ is carried by the cable whereas the remaining part $p-p_{1}$ is carried by the bending stiffness of the beam. In this case, it is well known $[11,14,17]$ that the equation for the (downwards) displacement $w$ of the beam is

$$
E I w^{\prime \prime \prime \prime}(x)=p(x)-p_{1}(x) \quad \forall x \in(0, L) .
$$

The horizontal tension of the cable is increased to $H+h(w)$ and the deflection $w$ is added to the displacement $y$. Hence, according to (2.1), the equation which takes into account this condition reads

$$
(H+h(w))\left(y^{\prime \prime}(x)+w^{\prime \prime}(x)\right)=-q-p_{1}(x) \quad \forall x \in(0, L) .
$$

Then, combining (2.3), (2.4) and (2.5) we obtain

$$
E I w^{\prime \prime \prime \prime}(x)-(H+h(w)) w^{\prime \prime}(x)+\frac{q}{H} h(w)=p(x) \quad \forall x \in(0, L),
$$

which is known in literature as the Melan equation [17, p. 77]. The beam representing the bridge is assumed to be hinged at its endpoints, which means that the boundary conditions to be associated with (2.6) read

$$
w(0)=w(L)=w^{\prime \prime}(0)=w^{\prime \prime}(L)=0 .
$$

Equation (2.6) is by far nontrivial: it is a nonlinear integrodifferential equation of fourth order. A further simplification is to consider $h$ as a small constant (see, e.g., $[6,(4.10)])$ and obtain the linear equation

$$
E I w^{\prime \prime \prime \prime}(x)-(H+h) w^{\prime \prime}(x)=p(x)-\frac{h q}{H} \quad \forall x \in(0, L),
$$

which can be integrated with classical methods. In the engineering literature, (2.6) and its simplifications have been used for the computation of moments and shears for different kinds of suspension bridges; see [17, 26].

\section{How to compute the additional tension}

In this section we address the problem of the computation of the additional tension $h=h(w)$ in (2.6). Since the cable is extensible, it may be that $h(w) \neq 0$. To fix the ideas, we first recall that the sag-span ratio is around $1 / 10$, see e.g. [22, Section 15.17]; by using both (2.2) and (2.3), this means that

$$
y\left(\frac{L}{2}\right)-y(0)=\frac{L}{10} \Longrightarrow \frac{q}{H}=\frac{4}{5 L} \Longrightarrow y^{\prime}(0)=0.4 .
$$


The length $L_{c}$ of the cable at rest is given by

$$
L_{c}=\int_{0}^{L} \sqrt{1+y^{\prime}(x)^{2}} d x=\frac{L}{2} \sqrt{1+\frac{L^{2} q^{2}}{4 H^{2}}}+\frac{H}{q} \log \left(\frac{L q}{2 H}+\sqrt{1+\frac{L^{2} q^{2}}{4 H^{2}}}\right) .
$$

If we assume (3.1), then $L_{c}$ may be written as a linear function of $L$ :

$$
L_{c}=\left(\frac{\sqrt{29}}{10}+\frac{5}{4} \log \frac{2+\sqrt{29}}{5}\right) L \approx 1.026 L .
$$

The increase $\Delta L_{c}$ of the length $L_{c}$ due to the deformation $w$ is

$$
\Delta L_{c}=\Gamma(w):=\int_{0}^{L}\left(\sqrt{1+\left[y^{\prime}(x)+w^{\prime}(x)\right]^{2}}-\sqrt{1+y^{\prime}(x)^{2}}\right) d x .
$$

According to (2.3) and (3.3), the exact value of $\Gamma(w)$ is

$$
\Gamma(w)=\int_{0}^{L} \sqrt{1+\left[w^{\prime}(x)+\frac{q}{H}\left(\frac{L}{2}-x\right)\right]^{2}} d x-L_{c} .
$$

Finally, if $A$ denotes the cross-sectional area of the cable and $E$ denotes the modulus of elasticity of the material, then the additional tension in the cable produced by the live load $p$ is given by

$$
h=\frac{E A}{L_{c}} \Delta L_{c}, \quad h(w)=\frac{E A}{L_{c}} \Gamma(w) .
$$

In literature, there are at least three different ways to approximate $\Gamma(w)$. Let us analyze them in detail.

First approximation. Recall the asymptotic expansion, valid for any $\rho \neq 0$,

$$
\sqrt{1+(\rho+\varepsilon)^{2}}-\sqrt{1+\rho^{2}} \sim \frac{\varepsilon \rho}{\sqrt{1+\rho^{2}}} \quad \text { as } \varepsilon \rightarrow 0 .
$$

By applying it to (3.4) one obtains

$$
\Delta L_{c} \approx \int_{0}^{L} \frac{y^{\prime}(x) w^{\prime}(x)}{\sqrt{1+y^{\prime}(x)^{2}}} d x .
$$

While introducing the model in Figure 1, von Kármán and Biot [14, p. 277] warned the reader by writing

whereas the deflection of the beam may be considered small, the deflection of the string, i.e., the deviation of its shape from a straight line, has to be considered as of finite magnitude.

However, after reaching (3.7), von Kármán and Biot [14, (5.14)] decided to neglect $y^{\prime}(x)^{2}$ in comparison with unity and wrote

$$
\begin{aligned}
\Gamma(w) & \approx \Gamma_{1}(w)=\int_{0}^{L} y^{\prime}(x) w^{\prime}(x) d x \\
& =-\int_{0}^{L} w(x) y^{\prime \prime}(x) d x=\frac{q}{H} \int_{0}^{L} w(x) d x,
\end{aligned}
$$


where the integration by parts takes into account that $w(0)=w(L)=0$ and, for the second equality, one uses (2.3). We denote by $\Gamma_{1}$ the approximated quantity obtained in [14]. A first approximation of $\Gamma(w)$ is then

$$
\Gamma_{1}(w)=\frac{q}{H} \int_{0}^{L} w(x) d x .
$$

Assuming that $y^{\prime}(x)$ is small means that the cable is almost horizontal, which seems quite far from the truth. This is a mistake while deriving (3.8): it was already present in the Report [2, Section VI-5] and also appears in more recent literature; see $[30,(17)]$ and $[7,(1)]$.

In order to quantify the error of this approximation, we notice that (3.1) yields

$$
\sqrt{1+y^{\prime}(0)^{2}} \approx 1.077
$$

yielding an error of $7.7 \%$ if we approximate with unity. The same error occurs at the other endpoint $(x=L)$. Using again (3.1), a similar computation leads to

$$
\sqrt{1+y^{\prime}\left(\frac{L}{4}\right)^{2}} \approx 1.02
$$

yielding an error of $2 \%$, while it is clear that there is no error at all at the vertex of the parabola $x=L / 2$. In some particular situations one may also have a sag-span ratio of $1 / 8$, in which case

$$
y^{\prime}(0)=\frac{1}{2} \text { and } \sqrt{1+y^{\prime}(0)^{2}} \approx 1.12,
$$

yielding an error of $12 \%$. In any case, this approximation appears too rude.

Second approximation. After reaching (3.3), Timoshenko [27, 28] (see also [29, Chapter 11]) multiplied and divided the integrand by its conjugate expression and obtained

$$
\Gamma(w)=\int_{0}^{L} \frac{2 w^{\prime}(x) y^{\prime}(x)+w^{\prime}(x)^{2}}{\sqrt{1+\left[y^{\prime}(x)+w^{\prime}(x)\right]^{2}}+\sqrt{1+y^{\prime}(x)^{2}}} d x .
$$

Then he neglected the derivatives and approximated the denominator with 2 :

$$
\Gamma(w) \approx \int_{0}^{L}\left(w^{\prime}(x) y^{\prime}(x)+\frac{w^{\prime}(x)^{2}}{2}\right) d x .
$$

With an integration by parts and taking into account both $w(0)=w(L)=0$ and (2.3) we obtain

$$
\Gamma_{2}(w)=\frac{q}{H} \int_{0}^{L} w(x) d x+\int_{0}^{L} \frac{w^{\prime}(x)^{2}}{2} d x .
$$

With two further integrations by parts one may also obtain (see [29, (11.16)])

$$
\Gamma_{2}(w)=\frac{q}{H} \int_{0}^{L} w(x) d x-\frac{1}{2} \int_{0}^{L} w(x) w^{\prime \prime}(x) d x,
$$


but we prefer to stick to (3.9) since it does not involve the second derivative of $w$. Note that also $\Gamma_{2}$ is obtained by neglecting $y^{\prime}$ which, as already underlined, is not small compared to unity, especially near the endpoints $x=0$ and $x=L$.

Third approximation. Without neglecting $y^{\prime}$, an integration by parts and the conditions $w(0)=w(L)=0$ transform (3.7) into

$$
\Delta L_{c} \approx-\int_{0}^{L} \frac{y^{\prime \prime}(x) w(x)}{\left(1+y^{\prime}(x)^{2}\right)^{3 / 2}} d x .
$$

Hence, invoking (2.3), a third approximation of $\Gamma$ is

$$
\Gamma_{3}(w)=\frac{q}{H} \int_{0}^{L} \frac{w(x)}{\left[1+\frac{q^{2}}{H^{2}}\left(x-\frac{L}{2}\right)^{2}\right]^{3 / 2}} d x
$$

In order to obtain (3.10), one uses the asymptotic expansion (3.6) which holds for any $\rho \neq 0$ and for $|\varepsilon| \ll|\rho|$. But, in our case, from (2.3) we have that $\rho=y^{\prime}(x)$ and hence $\rho=0$ if $x=\frac{L}{2}$. More generally, since $y$ is given and $w$ depends on the load $p,\left|w^{\prime}(x)\right|$ may not be small when compared to $\left|y^{\prime}(x)\right|$. So, a second mistake is that (3.6) is not correct for any $x \in(0, L)$. Nevertheless, if the live load $p=p(x)$ is assumed to be symmetric with respect to $x=\frac{L}{2}$ (the center of the beam) also the displacement $w$ will have such symmetry and then $\left|w^{\prime}(x)\right|$ will indeed be small with respect to $\left|y^{\prime}(x)\right|$ for all $x$; in particular,

$$
w^{\prime}\left(\frac{L}{2}\right)=y^{\prime}\left(\frac{L}{2}\right)=0 .
$$

Hence, this approximation appears reasonable only if the live load $p$ is "almost" symmetric.

Note that $\Gamma_{2}$ equals $\Gamma_{1}$ plus an additional positive term and that $\Gamma_{3}$ has a smaller integrand when compared to $\Gamma_{1}$; therefore,

$$
\Gamma_{3}(w)<\Gamma_{1}(w)<\Gamma_{2}(w) \quad \forall w .
$$

In the next sections we compare (3.4), (3.8), (3.9) and (3.10) and we show that there may be large discrepancies.

\section{Some explicit computations}

In this section we estimate the difference of behaviors of $\Gamma_{i}$ for some particular vertical displacements $w$. To this end, we notice that it is likely to expect that the maximum vertical displacement of the beam is around 1/100 of the length of the span; if the bridge is $1 \mathrm{~km}$ long, the maximum amplitude of the vertical oscillation should be expected of at most $10 \mathrm{~m}$. Whence, a reasonable assumption is that

$$
w\left(\frac{L}{2}\right)=\frac{L}{100} .
$$

We now compute the $\Gamma_{i}$ 's on three different configurations of the beam. 
Parabolic shape. Assume the displacement $w$ has the shape of a parabola,

$$
w(x)=\delta x(L-x) \quad(\delta>0),
$$

although this does not represent a hinged beam since it fails to satisfy the conditions $w^{\prime \prime}(0)=w^{\prime \prime}(L)=0$. However, this simple case allows for hand computations and gives a qualitative idea of the differences between $\Gamma$ and its approximations $\Gamma_{i}(i=1,2,3)$. For the configuration (4.2), the constraint (4.1) implies that

$$
\delta=\frac{1}{25 L} .
$$

Let $w$ be as in (4.2), then (3.4), (3.8), (3.9), (3.10), combined with (3.1) and (4.3), yield

$$
\begin{aligned}
\Gamma(w) & =\left[\frac{\sqrt{746}-5 \sqrt{29}}{50}+\frac{25}{22} \log \frac{11+\sqrt{746}}{25}-\frac{5}{4} \log \frac{2+\sqrt{29}}{5}\right] L, \\
\Gamma_{1}(w) & =\frac{2}{375} L, \quad \Gamma_{2}(w)=\frac{7}{1250} L=\frac{21}{20} \Gamma_{1}(w), \\
\Gamma_{3}(w) & =\left[\frac{\sqrt{29}}{4}-\frac{25}{16} \log \frac{33+4 \sqrt{29}}{25}\right] \frac{L}{25} .
\end{aligned}
$$

Whence, if $w$ is as in (4.2) and we assume both (3.1) and (4.3), then

$$
\Gamma_{1}(w) \approx \Gamma(w), \quad \Gamma_{2}(w) \approx 1.05 \Gamma(w), \quad \Gamma_{3}(w) \approx 0.96 \Gamma(w) .
$$

Simplest symmetric beam shape. The simplest shape for a hinged beam is the fourth-order polynomial

$$
w(x)=\delta x\left(x^{3}-2 L x^{2}+L^{3}\right) \quad(\delta>0) ;
$$

this function will also serve to build Counterexample 1. In this case, if we assume again (4.1), we obtain

$$
\delta=\frac{4}{125 L^{3}} .
$$

Inserting (3.1) and (4.5) into (3.4) and using $w$ as in (4.4), a numerical computation with Mathematica gives

$$
\Gamma(w) \approx 0.00512 L .
$$

In turn, by inserting (4.4) into (3.8), (3.9), (3.10) and by using (3.1) and (4.5) we find

$$
\begin{aligned}
& \Gamma_{1}(w)=\frac{16}{3125} L, \\
& \Gamma_{2}(w)=\frac{2808}{546875} L, \\
& \Gamma_{3}(w)=\left[\frac{23}{5} \sqrt{29}-\frac{123}{4} \log \frac{33+4 \sqrt{29}}{25}\right] \frac{L}{160} .
\end{aligned}
$$


Therefore,

$$
\Gamma_{1}(w) \approx \Gamma_{2}(w) \approx \Gamma(w) \approx 1.05 \Gamma_{3}(w)
$$

Asymmetric beams. We assume here that there is some load concentrated on the interval $(0, \ell)$ for some $\ell \in\left(0, \frac{L}{2}\right)$ (the case $\ell>\frac{L}{2}$ being specular) and that the corresponding deformation $w$ has the shape of the piecewise affine function

$$
w(x)= \begin{cases}\sigma x & \text { if } x \in(0, \ell), \\ \frac{\sigma \ell}{L-\ell}(L-x) & \text { if } x \in(\ell, L),\end{cases}
$$

so that $w(\ell)=\sigma \ell$. A reasonable value of $\sigma$ satisfies the rule in (4.1), that is,

$$
\sigma \ell=w(\ell)=\frac{\ell}{50} \Longrightarrow \sigma=\frac{1}{50} \text {. }
$$

Inserting (4.6) into (3.4) and using both (3.1) and (4.7) ( $\Gamma$ is not linear with respect to $\sigma$ ) we find the formula

$$
\begin{aligned}
\Gamma(w)=[ & \Phi\left(\frac{4 \ell}{5 L}-\frac{21}{50}\right)-\Phi\left(-\frac{21}{50}\right)+\Phi\left(\frac{2}{5}+\frac{1}{50} \frac{\ell}{L-\ell}\right) \\
& \left.-\Phi\left(\frac{4 \ell}{5 L}-\frac{2}{5}+\frac{1}{50} \frac{\ell}{L-\ell}\right)-2 \Phi\left(\frac{2}{5}\right)\right] \frac{5}{8} L
\end{aligned}
$$

where we also used (3.2) and

$$
\Phi(s)=s \sqrt{1+s^{2}}+\log \left(s+\sqrt{1+s^{2}}\right) .
$$

Some tedious computations show that

$$
\begin{aligned}
\Gamma(w) \rightarrow & {\left[\frac{21 \sqrt{2941}-\sqrt{2501}}{1250}-\frac{4 \sqrt{29}}{25}\right.} \\
& \left.+2 \log \frac{(\sqrt{2501}-1)(\sqrt{2941}+21)}{1250(2+\sqrt{29})}\right] \frac{5}{8} L \quad \text { as } \ell \rightarrow \frac{L}{2}, \\
\Gamma(w) \sim & \frac{\sqrt{2941}-10 \sqrt{29}}{50} \ell \text { as } \ell \rightarrow 0 .
\end{aligned}
$$

Inserting (4.6) into (3.8), (3.9) and (3.10) and using (3.1) we find

$$
\begin{aligned}
& \Gamma_{1}(w)=\frac{2 \ell}{5} \sigma \\
& \Gamma_{2}(w)=\frac{2 \ell}{5} \sigma+\frac{L \ell}{L-\ell} \frac{\sigma^{2}}{2}, \\
& \Gamma_{3}(w)=\left(\frac{\sqrt{29}}{4} L-\sqrt{\frac{29}{16} L^{2}+\ell^{2}-L \ell}\right) \frac{\sigma L}{L-\ell} .
\end{aligned}
$$

Both $\Gamma_{1}$ and $\Gamma_{3}$ linearly depend on $\sigma$. Summarizing, in the asymmetric case we find that

$$
\frac{\Gamma_{1}(w)}{\Gamma(w)} \rightarrow 1.054, \quad \frac{\Gamma_{2}(w)}{\Gamma(w)} \rightarrow 1.08, \quad \frac{\Gamma_{3}(w)}{\Gamma(w)} \rightarrow 1.015 \quad \text { as } \ell \rightarrow 0
$$


yielding approximate errors of $5.4 \%, 8 \%, 1.5 \%$, respectively. Moreover,

$$
\frac{\Gamma(w)}{\Gamma_{1}(w)} \rightarrow 1.008, \quad \frac{\Gamma(w)}{\Gamma_{2}(w)} \rightarrow 0.96, \quad \frac{\Gamma(w)}{\Gamma_{3}(w)} \rightarrow 1.047 \quad \text { as } \ell \rightarrow \frac{L}{2},
$$

yielding approximate errors of $0.8 \%, 4 \%, 4.7 \%$, respectively.

\section{Existence and uniqueness results}

Here and in what follows we denote the $L^{p}$-norms by

$$
\|v\|_{p}:=\|v\|_{L^{p}(0, L)} \quad \forall p \in[1, \infty], \forall v \in L^{p}(0, L) .
$$

In this section we prove the existence of at least one solution of (2.6)-(2.7). For simplicity, we drop some constants and consider the problem

$$
\begin{gathered}
w^{\prime \prime \prime \prime}(x)-(a+h(w)) w^{\prime \prime}(x)+b h(w)=p(x) \quad \text { for } x \in(0, L), \\
w(0)=w(L)=w^{\prime \prime}(0)=w^{\prime \prime}(L)=0,
\end{gathered}
$$

where $a, b>0$ and $h(w)$ is a nonlocal term, of indefinite sign, satisfying

$$
\exists c>0, \quad|h(u)| \leq c\|u\|_{1} \quad \forall u \in H_{0}^{1}(0, L) .
$$

Note that assumption (5.2) is satisfied when $h$ is defined by

$$
h(w)=\frac{E A}{L_{c}} \Gamma_{i}(w) \quad(i=1,3),
$$

see (3.5), with $\Gamma_{1}$ and $\Gamma_{3}$ defined in (3.8) and (3.10). In both these cases, one can take

$$
c=\frac{E A}{L_{c}} \frac{q}{H} .
$$

Our first results yields the existence of a solution of (5.1) provided that $L$ and $p$ are sufficiently small.

Theorem 5.1. Let $a, b>0$ and let $h: H_{0}^{1}(0, L) \rightarrow \mathbb{R}$ be a continuous functional such that there exists $c>0$ satisfying (5.2). Assume that

$$
L^{5}<\frac{\pi^{3}}{b c}
$$

Then for all $p \in L^{1}(0, L)$ satisfying

$$
\|p\|_{1} \leq \frac{a\left(\pi^{3}-b c L^{5}\right)}{c L^{4}}
$$

there exists at least one solution $w \in W^{4,1}(0, L) \cap H_{0}^{1}(0, L)$ of (5.1) which satisfies the estimate

$$
\|w\|_{\infty} \leq \frac{L^{3}}{\pi^{3}-b c L^{5}}\|p\|_{1} .
$$


We prove Theorem 5.1 in Section 8. Theorem 5.1 does not apply to $\Gamma$ since the corresponding function $h$ in (3.5) fails to satisfy (5.2). So, we now state a different result which allows to include $\Gamma$.

Consider again (5.1) with $a, b>0$ and $h(w)$ being a nonlocal term, of indefinite sign, satisfying

$$
\exists c>0, \quad|h(u)| \leq c\left\|u^{\prime}\right\|_{1} \quad \forall u \in H_{0}^{1}(0, L) .
$$

Note that assumption (5.5) is satisfied when $h$ is defined by

$$
h(w)=\frac{E A}{L_{c}} \Gamma(w),
$$

see (3.5), with $\Gamma$ defined in (3.4). Indeed, from the simple inequality

$$
\sqrt{1+(\gamma+s)^{2}}-\sqrt{1+\gamma^{2}} \leq|s| \quad \forall \gamma \in \mathbb{R}, \forall s \in \mathbb{R},
$$

we infer that

$$
|\Gamma(w)| \leq \int_{0}^{L}\left|\sqrt{1+\left[y^{\prime}(x)+w^{\prime}(x)\right]^{2}}-\sqrt{1+y^{\prime}(x)^{2}}\right| d x \leq \int_{0}^{L}\left|w^{\prime}(x)\right| d x,
$$

and therefore one can take $c=1$ in (5.5). In Section 9 we prove the following theorem.

Theorem 5.2. Let $a, b>0$ and let $h: H_{0}^{1}(0, L) \rightarrow \mathbb{R}$ be a continuous functional such that there exists $c>0$ satisfying (5.5). Assume that

$$
L^{4}<\frac{1}{b c}
$$

Then for all $p \in L^{1}(0, L)$ satisfying

$$
\|p\|_{1} \leq \frac{a\left(1-b c L^{4}\right)}{c L^{3}},
$$

there exists at least one solution $w \in W^{4,1}(0, L) \cap H_{0}^{1}(0, L)$ of (5.1) which satisfies the estimate

$$
\left\|w^{\prime}\right\|_{\infty} \leq \frac{L^{2}}{1-b c L^{4}}\|p\|_{1} .
$$

Remark 5.3. Neither Theorem 5.1 nor Theorem 5.2 covers the case where $h$ is defined through $\Gamma_{2}$ since

$$
\left|\Gamma_{2}(w)\right| \leq c\|w\|_{1}+\frac{\left\|w^{\prime}\right\|_{2}^{2}}{2} \quad \forall w \in H_{0}^{1}(0, L),
$$

and therefore $\Gamma_{2}$ has quadratic growth. However, using some a priori bounds for the linearized equation, one may estimate the quadratic term $\left\|w^{\prime}\right\|_{2}^{2}$ with a linear term $\left\|w^{\prime}\right\|_{2}$ and, consequently, obtain a result in the spirit of Theorems 5.1 and 5.2 also when $h$ is defined through $\Gamma_{2}$. However, we will not pursue this here.

So far, we merely stated existence results for small solutions of (5.1). We now prove an existence and uniqueness result (for small solutions) which, 
however, has the disadvantage of some tedious and painful assumptions. We first assume that

$$
\begin{array}{r}
h(0)=0, \quad \exists c>0, \quad|h(u)-h(v)| \leq c\left\|u^{\prime \prime}-v^{\prime \prime}\right\|_{2} \\
\forall u, v \in H^{2}(0, L) \cap H_{0}^{1}(0, L) .
\end{array}
$$

When $h$ is defined by (3.5), condition (5.8) is satisfied for $\Gamma, \Gamma_{1}$ or $\Gamma_{3}$.

In Section 10 we prove the following existence and uniqueness result for small solutions of (5.1) which, again, holds when both $L$ and $p$ are sufficiently small.

Theorem 5.4. Let $a, b>0$ and let $h: H_{0}^{1}(0, L) \rightarrow \mathbb{R}$ be a continuous functional such that there exists $c>0$ satisfying (5.8). Assume that

$$
L<\min \left\{\frac{1}{(b c)^{2}}, \frac{\pi}{(b c)^{2 / 5}}\right\} .
$$

Then for all $p \in L^{1}(0, L)$ satisfying

$$
\|p\|_{1}<\min \left\{\left(\frac{\pi}{L}\right)^{3 / 2} \frac{\left(\pi^{5 / 2}-b c L^{5 / 2}\right)(1-b c \sqrt{L})}{c\left(\pi^{5 / 2}-b c L^{5 / 2}+b c \pi L^{7 / 2}\right)}, \frac{a\left(\pi^{5 / 2}-b c L^{5 / 2}\right)}{\pi c L^{5 / 2}}\right\},
$$

there exists a unique solution $w \in W^{4,1}(0, L) \cap H_{0}^{1}(0, L)$ of $(5.1)$ satisfying

$$
\left\|w^{\prime \prime}\right\|_{2} \leq \frac{\pi L^{5 / 2}}{\pi^{5 / 2}-b c L^{5 / 2}}\|p\|_{1}
$$

Note that the smallness of $L$ assumed in (5.9) ensures that the righthand side of (5.10) is positive. Clearly, which is the maximum to be considered in (5.9) depends on whether $b c \lessgtr 1$. We also emphasize that Theorem 5.4 only states the existence and uniqueness of a small solution satisfying (5.11), but it does not clarify if there exist additional large solutions violating (5.11). And, indeed, as the following counterexample shows, there may exist additional large solutions and, hence, Theorem 5.4 cannot be improved without further assumptions.

Counterexample 1. For a given $L>\sqrt{12}$ consider the functional

$$
h(w)=\int_{0}^{L} w^{\prime \prime}(x) d x \quad \forall w \in H^{2}(0, L) \cap H_{0}^{1}(0, L)
$$

so that (5.8) is satisfied with $c=\sqrt{L}$. Fix $\delta>0$, for instance as in (4.5), and consider the problem

$$
\begin{gathered}
w^{\prime \prime \prime \prime}(x)-\left(2 \delta L^{3}+\varepsilon+h(w)\right) w^{\prime \prime}(x)+\frac{12}{L^{3}} h(w)=p_{\varepsilon}(x) \text { for } x \in(0, L), \\
w(0)=w(L)=w^{\prime \prime}(0)=w^{\prime \prime}(L)=0,
\end{gathered}
$$

where $p_{\varepsilon}(x)=12 \delta \varepsilon\left(L x-x^{2}\right)$ and $\varepsilon>0$ will be fixed later. Equation (5.12) is 
as (5.1) with

$$
a=2 \delta L^{3}+\varepsilon, \quad b=\frac{12}{L^{3}}, \quad c=\sqrt{L}, \quad p(x)=p_{\varepsilon}(x) .
$$

Whence,

$$
\frac{1}{(b c)^{2}}=\frac{L^{5}}{144}, \quad \frac{\pi}{(b c)^{2 / 5}}=\frac{\pi L}{12^{2 / 5}} .
$$

Since we assumed that $L>\sqrt{12}$ and since $12^{2 / 5}<\pi$, condition (5.9) is satisfied. Now we choose $\varepsilon>0$ sufficiently small so that $p_{\varepsilon}$ satisfies the bound (5.10). Then all the assumptions of Theorem 5.4 are fulfilled and there exists a unique solution $w$ of (5.12) satisfying (5.11).

Note that the function $w_{\delta}(x)=\delta x\left(x^{3}-2 L x^{2}+L^{3}\right)$, already considered in (4.4), solves (5.12). However, if $\varepsilon>0$ is sufficiently small, it fails to satisfy (5.11) and therefore $w_{\delta}$ is not the small solution found in Theorem 5.4. This shows that, besides a small solution, also a large solution may exist.

We conclude this section with a simple calculus statement which will be repeatedly used in what follows, both for proving the above statements through a fixed point argument and for implementing the numerical procedures.

Proposition 5.5. Let $\alpha>0$ and $f \in L^{1}(0, L)$. The unique solution

$$
u \in W^{4,1}(0, L) \cap H_{0}^{1}(0, L)
$$

of the problem

$$
\begin{gathered}
u^{\prime \prime \prime \prime}(x)-\alpha^{2} u^{\prime \prime}(x)=f(x) \quad \text { in }(0, L), \\
u(0)=u(L)=u^{\prime \prime}(0)=u^{\prime \prime}(L)=0
\end{gathered}
$$

is given by

$$
\begin{aligned}
u(x)= & \frac{x}{\alpha^{2} L} \int_{0}^{L}(L-t) f(t) d t-\frac{\sinh (\alpha x)}{\alpha^{3} \sinh (\alpha L)} \int_{0}^{L} \sinh [\alpha(L-t)] f(t) d t \\
& +\int_{0}^{x}\left[\frac{t-x}{\alpha^{2}}+\frac{\sinh [\alpha(x-t)]}{\alpha^{3}}\right] f(t) d t .
\end{aligned}
$$

Note that the assumption $\alpha^{2}>0$ in Proposition 5.5 is crucial since otherwise the equation changes type: instead of hyperbolic functions one has trigonometric functions with possible resonance problems.

\section{Numerical implementations with a stable fixed point}

In this section and the following one we apply an iterative procedure in order to numerically determine a solution of (5.1). We inductively construct sequences $\left\{w_{n}\right\}$ of approximating solutions and it turns out that an excellent estimator of the rate of approximation is the corresponding numerical sequence $\left\{h\left(w_{n}\right)\right\}$. As we shall see, depending on the parameters involved, the fixed points of our iterative methods may be stable or unstable. In this 
section we deal with stable cases whereas in Section 7, which involves an actual bridge, we deal with an unstable case.

We drop here the constant $E A / L_{c}$ so that $h(w)=\Gamma(w)$, we fix constants $a, b, c>0$ and a load $p$, and consider the equations

$$
a w^{\prime \prime \prime \prime}(x)-(b+h(w)) w^{\prime \prime}(x)+c h(w)=p(x) \quad \forall x \in(0, L),
$$

complemented with the boundary conditions (2.7). We define a map $\Lambda: \mathbb{R} \rightarrow$ $\mathbb{R}$ as follows. For any $\Theta \in \mathbb{R}$ we denote by $W_{\Theta}$ the unique solution of the equation

$$
a w^{\prime \prime \prime \prime}(x)-(b+\Theta) w^{\prime \prime}(x)+c \Theta=p(x) \quad \forall x \in(0, L),
$$

satisfying (2.7). The solution of this equation may be obtained by using Proposition 5.5. Then we put

$$
\Lambda(\Theta):=h\left(W_{\Theta}\right) .
$$

Clearly, $W_{\Theta}$ is a solution of (6.1) and (2.7) if and only if $\Theta$ is a fixed point for $\Lambda$, that is, $h\left(W_{\Theta}\right)=\Lambda(\Theta)=\Theta$.

If $\Lambda(\Theta) \neq \Theta$, we can hope to find the fixed point for $\Lambda$ by an iterative procedure. We fix some $\Theta_{0} \in \mathbb{R}$ (for instance, $\Theta_{0}=0$ ) and define a sequence $\Theta_{n}:=\Lambda\left(\Theta_{n-1}\right)$ for all $n \geq 1$. This defines a discrete dynamical system which, under suitable conditions, may force the sequence to converge to the fixed point $\bar{\Theta}$ of $\Lambda$. For the equations considered in this section, this procedure works out perfectly.

In the tables below we report some of our numerical results; we always start with $\Theta_{0}=0$. For each table we emphasize the values of the parameters involved in (6.1). Since $\bar{\Theta}$ turned out to be small, we magnify $\Lambda\left(\Theta_{n}\right)$ by some powers of 10 .

TABle 1 . The case $L=2, a=b=c=1, p(x) \equiv 1$.

\begin{tabular}{|c|c|c|c|c|c|c|c|c|}
\hline$n$ & 1 & 2 & 3 & 4 & 5 & 6 & 7 & 8 \\
\hline $100 \Lambda\left(\Theta_{n}\right)$ & 9.55239 & 8.1815 & 8.37021 & 8.34408 & 8.3477 & 8.3472 & 8.34727 & 8.34726 \\
\hline
\end{tabular}

TABle 2. The case $L=2, a=b=c=1, p(x)=0$ in $(0,1)$ and $p(x)=10$ in $(1,2)$.

\begin{tabular}{|c|c|c|c|c|c|c|c|c|}
\hline$n$ & 1 & 2 & 3 & 4 & 5 & 6 & 7 & 8 \\
\hline $10 \Lambda\left(\Theta_{n}\right)$ & 8.04928 & 4.80539 & 5.90186 & 5.50443 & 5.6451 & 5.59488 & 5.61276 & 5.60638 \\
\hline
\end{tabular}

TABlE 3 . The case $L=2, a=b=c=1, p(x)=0$ in $(0,3 / 2)$ and $p(x)=20$ in $(3 / 2,2)$.

\begin{tabular}{|c|c|c|c|c|c|c|c|c|}
\hline$n$ & 1 & 2 & 3 & 4 & 5 & 6 & 7 & 8 \\
\hline $10 \Lambda\left(\Theta_{n}\right)$ & 3.93699 & 2.84652 & 3.1149 & 3.04668 & 3.06388 & 3.05954 & 3.06064 & 3.06036 \\
\hline
\end{tabular}


TABLE 4 . The case $L=2, a=b=c=1, p(x)=10 e^{-10(x-1)^{2}}$.

\begin{tabular}{|c|c|c|c|c|c|c|c|c|}
\hline$n$ & 1 & 2 & 3 & 4 & 5 & 6 & 7 & 8 \\
\hline $10 \Lambda\left(\Theta_{n}\right)$ & 6.19365 & 3.96853 & 4.65526 & 4.4316 & 4.50324 & 4.48017 & 4.48758 & 4.4852 \\
\hline
\end{tabular}

TABle 5 . The case $L=2, a=10, b=c=1, p(x) \equiv 1$.

\begin{tabular}{|c|c|c|c|c|}
\hline$n$ & 1 & 2 & 3 & 4 \\
\hline $100 \Lambda\left(\Theta_{n}\right)$ & 1.02565 & 1.01427 & 1.01439 & 1.01439 \\
\hline
\end{tabular}

TABLE 6 . The case $L=2, a=b=1, c=10, p(x) \equiv 1$.

\begin{tabular}{|c|c|c|c|c|c|c|c|c|}
\hline$n$ & 1 & 2 & 3 & 4 & 5 & 6 & 7 & 8 \\
\hline $100 \Lambda\left(\Theta_{n}\right)$ & 9.55239 & 0.3214 & 9.16847 & 0.60499 & 8.83393 & 0.858085 & 8.5387 & 1.08607 \\
\hline
\end{tabular}

In all the above results it appears that the sequence $\left\{\Lambda\left(\Theta_{n}\right)\right\}$ is not monotonic but the two subsequences of odd and even iterations appear, respectively, decreasing and increasing. Moreover, since they converge to the same limit, this means that

$$
\Lambda\left(\Theta_{2 k}\right)<\Lambda\left(\Theta_{2 k+2}\right)<\bar{\Theta}<\Lambda\left(\Theta_{2 k+1}\right)<\Lambda\left(\Theta_{2 k-1}\right) \quad \forall k \geq 1 .
$$

This readily gives an approximation of $\bar{\Theta}$ and, in turn, of the solution $\bar{w}$ of (6.1). As should be expected, the convergence is slower for larger values of $c$ : in the very last experiment we found

$$
100 \Lambda\left(\Theta_{126}\right)<4.3 \text { and } 100 \Lambda\left(\Theta_{127}\right)>4.7 .
$$

In all these cases this procedure worked out, which means that the fixed point $\bar{\Theta}$ is stable and that the discrete dynamical system may be described as in Figure 2. The map $\Theta \mapsto \Lambda(\Theta)$ is decreasing and its slope is larger than -1 in a neighborhood of $\bar{\Theta}$.

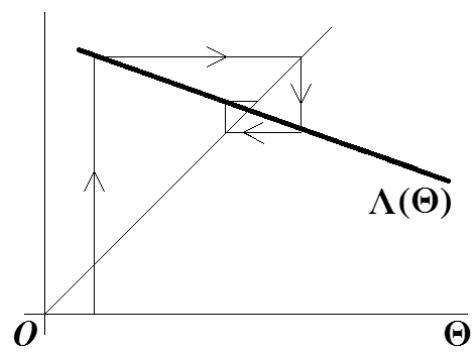

Figure 2. The stable fixed point for the map $\Theta \mapsto \Lambda(\Theta)$ defined by (6.2).

We also used this iterative procedure in order to estimate the responses of the different forms of $h=\Gamma_{i}$. We fix the parameters involved in (6.1) 
and we perform the iterative procedure for each one of the $\Gamma_{i}(i=1,2,3)$ and $\Gamma_{0}=\Gamma$. We define again $\Lambda_{i}(\Theta)(i=0,1,2,3)$ as in (6.2). After a finite number of iterations we have a good approximation of

$$
\bar{\Theta}_{i}:=\lim _{n \rightarrow \infty} \Lambda_{i}\left(\Theta_{n}\right) .
$$

Then, we obtain a limit equation (6.1) having the form

$$
a w^{\prime \prime \prime \prime}(x)-\left(b+\bar{\Theta}_{i}\right) w^{\prime \prime}(x)+c \bar{\Theta}_{i}=p(x) \quad \forall x \in(0, L),(i=0,1,2,3) .
$$

By integrating these linear equations with the boundary conditions (2.7) we obtain the different solutions. In Tables 7 and 8 we quote our numerical results for the different values of $\bar{\Theta}_{i}$.

TABLE 7 . The case $L=2, a=c=1, b=10, p(x) \equiv 1$.

\begin{tabular}{|c|c|c|c|}
\hline $100 \bar{\Theta}_{0}$ & $100 \bar{\Theta}_{1}$ & $100 \bar{\Theta}_{2}$ & $100 \bar{\Theta}_{3}$ \\
\hline 2.15633 & 2.07143 & 2.26845 & 1.98463 \\
\hline
\end{tabular}

TABle 8 . The case $L=2, a=c=1, b=10, p(x)=0$ in $(0,3 / 2)$ and $p(x)=20$ in $(3 / 2,2)$.

\begin{tabular}{|c|c|c|c|}
\hline $100 \bar{\Theta}_{0}$ & $100 \bar{\Theta}_{1}$ & $100 \bar{\Theta}_{2}$ & $100 \bar{\Theta}_{3}$ \\
\hline 7.7621 & 6.19506 & 8.47472 & 5.91363 \\
\hline
\end{tabular}

In all these experiments we found the same qualitative behavior represented in Figure 2: the sequence $\left\{\Lambda_{i}\left(\Theta_{n}\right)\right\}$ is not monotonic, it satisfies (6.3), and it converges to a fixed point for $\Lambda_{i}$. As we shall see in next section, this is not the case for different values of the parameters.

\section{Numerics with an unstable fixed point for an actual bridge}

We consider here a possible actual bridge and we fix the parameters in (2.6) following Wollmann [30]. The stiffness $E I$ is known to be $E I=57 \cdot 10^{6} \mathrm{kN} \cdot \mathrm{m}^{2}$ whereas $E A=36 \cdot 10^{8} \mathrm{kN}$. Wollmann considered a bridge with main span of length $L=460 \mathrm{~m}$ and he assumed (3.1) so that

$$
\frac{q}{H}=1.739 \cdot 10^{-3} \mathrm{~m}^{-1}, \quad q=170 \mathrm{kN} / \mathrm{m}, \quad H=97.75 \cdot 10^{3} \mathrm{kN} .
$$

By (3.2) we find $L_{c}=472 \mathrm{~m}$, while from (3.5) we infer that

$$
h(w)=\left(7.627 \cdot 10^{6} \mathrm{kN} / \mathrm{m}\right) \Gamma_{i}(w),
$$

where the $\Gamma_{i}(w)$ are measured in meters; we will consider $i=0,1,2,3$ with $\Gamma_{0}=\Gamma$ as in (3.4) and the remaining $\Gamma_{i}$ as in (3.8)-(3.10). 
We first take as live load a vehicle, a coach of length $10 \mathrm{~m}$ having a weight density of $10 \mathrm{kN} / \mathrm{m}$, that is,

$$
p(x)=10 \chi_{(d, d+10)} \mathrm{kN} / \mathrm{m} \quad 0<d<230,
$$

where $\chi_{(d, d+10)}$ denotes the characteristic function of the interval $(d, d+10)$. Then, after dropping the unity measure $\mathrm{kN} / \mathrm{m}$ and dividing by $10,(2.6)$ reads

$$
\begin{aligned}
57 \cdot 10^{5} w^{\prime \prime \prime \prime}(x) & -\left(9775+7.627 \cdot 10^{5} \Gamma_{i}(w)\right) w^{\prime \prime}(x) \\
+ & 1326 \Gamma_{i}(w)=\chi_{(d, d+10)} \quad \forall x \in(0,460),
\end{aligned}
$$

where the solution $w$ is computed in meters. For numerical reasons, it is better to rescale (7.1): we put

$$
w(x)=v\left(\frac{x}{230}\right)=v(s) .
$$

Let us compute the different values of $\Gamma_{i}$ after this change. We have

$$
\begin{aligned}
\Gamma_{0}(w) & =\int_{0}^{460} \sqrt{1+\left[w^{\prime}(x)+1.739 \cdot 10^{-3}(230-x)\right]^{2}} d x-1.026 \cdot 460 \\
& =230\left[\int_{0}^{2} \sqrt{1+\left[4.35 \cdot 10^{-3} v^{\prime}(s)+0.4(1-s)\right]^{2}} d s-2.052\right] \\
& =: \Upsilon_{0}(v) ; \\
\Gamma_{1}(w) & =1.739 \cdot 10^{-3} \int_{0}^{460} w(x) d x=0.4 \int_{0}^{2} v(s) d s=: \Upsilon_{1}(v) ; \\
\Gamma_{2}(w) & =0.4 \int_{0}^{2} v(s) d s+2.17 \cdot 10^{-3} \int_{0}^{2} v^{\prime}(s)^{2} d s=: \Upsilon_{2}(v) ; \\
\Gamma_{3}(w) & =1.739 \cdot 10^{-3} \int_{0}^{460} \frac{w(x) d x}{\left[1+3.02 \cdot 10^{-6}(x-230)^{2}\right]^{3 / 2}} \\
& =0.4 \int_{0}^{2} \frac{v(s) d s}{\left[1+0.16(s-1)^{2}\right]^{3 / 2}}=: \Upsilon_{3}(v) .
\end{aligned}
$$

Then, after the change (7.2) and division by

$$
\frac{57 \cdot 10^{5}}{230^{4}} \approx 2.037 \cdot 10^{-3}
$$

equation (7.1) becomes

$v^{\prime \prime \prime \prime}(s)-\left(90.72+7078 \Upsilon_{i}(v)\right) v^{\prime \prime}(s)+650999 \Upsilon_{i}(v)=491 \psi_{d}(s) \quad \forall s \in(0,2)$,

where $\psi_{d}$ is the characteristic function of the interval $\left(\frac{d}{230}, \frac{d+10}{230}\right)$. We try to proceed as in Section 6. We fix some $\Theta>0$ and we solve equation (7.3) by replacing $\Upsilon_{i}(v)$ with $\Theta$ :

$$
v^{\prime \prime \prime \prime}(s)-\alpha^{2} v^{\prime \prime}(s)=f(s) \quad \forall s \in(0,2),
$$

where

$$
\alpha^{2}:=90.72+7078 \Theta, \quad f(s):=491 \psi_{d}(s)-650999 \Theta .
$$


By Proposition 5.5, this linear equation, complemented with hinged boundary conditions, admits a unique solution $V_{\Theta}$ given by

$$
\begin{aligned}
V_{\Theta}(s)= & \left(\frac{491(455-d)}{10580}-650999 \Theta\right) \frac{s}{\alpha^{2}}+\frac{650999 \Theta}{2 \alpha^{2}} s^{2} \\
& +\frac{650999 \Theta}{\alpha^{4}}(1-\cosh (\alpha s)) \\
& +\left[650999 \Theta(\cosh (2 \alpha)-1)-982 \sinh \frac{\alpha}{46} \sinh \frac{\alpha(455-d)}{230}\right] \\
& \times \frac{\sinh (\alpha s)}{\alpha^{4} \sinh (2 \alpha)}+491 \Psi_{d, \Theta}(s),
\end{aligned}
$$

where

$$
\begin{aligned}
& \Psi_{d, \Theta}(s)= \\
& = \begin{cases}0 & \text { if } 0 \leq s \leq \frac{d}{230}, \\
\frac{1}{\alpha^{4}}\left(\cosh \left[\alpha\left(s-\frac{d}{230}\right)\right]-1\right)-\frac{\left(s-\frac{d}{230}\right)^{2}}{2 \alpha^{2}} & \text { if } \frac{d}{230}<s<\frac{d+10}{230}, \\
\frac{2}{\alpha^{4}} \sinh \frac{\alpha}{46} \sinh \left(\alpha\left(s-\frac{d+5}{230}\right)\right)+\frac{1}{46 \alpha^{2}}\left(\frac{d+5}{115}-2 s\right) & \text { if } \frac{d+10}{230} \leq s \leq 2 .\end{cases}
\end{aligned}
$$

We then compute $\Upsilon_{i}\left(V_{\Theta}\right)$ according to the above formulas and we put

$$
\Lambda_{i}(\Theta)=\Upsilon_{i}\left(V_{\Theta}\right) .
$$

Again, this defines a sequence $\Theta_{n}=\Lambda_{i}\left(\Theta_{n-1}\right)$. However, for the values in (7.3), this sequence appears to diverge and to be quite unstable: contrary to the experiments in Section 6, see (6.3), we have here that

$$
\Lambda_{i}\left(\Theta_{2 k}\right) \rightarrow+\infty, \quad \Lambda_{i}\left(\Theta_{2 k+1}\right) \rightarrow-\infty \quad \text { as } k \rightarrow \infty .
$$

This clearly describes an unstable fixed point, as represented in Figure 3. Here, the slope of $\Theta \mapsto \Lambda_{i}(\Theta)$ is smaller than -1 . In fact, our experiments show that it is very negative, possibly $-\infty$.

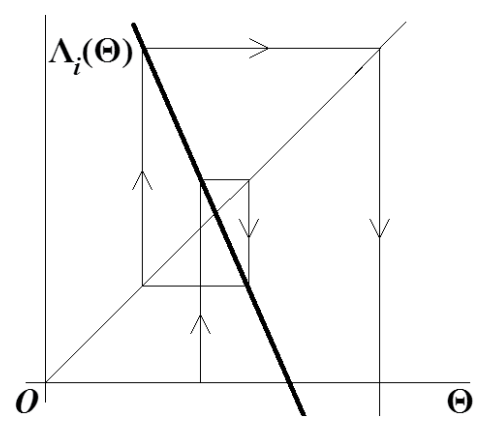

Figure 3. The unstable fixed point for the map $\Theta \mapsto \Lambda_{i}(\Theta)$ defined by (7.5). 
As already mentioned, in order to apply Proposition 5.5 one needs

$$
90.72+7078 \Theta_{n}>0
$$

since otherwise the equation changes type. These difficulties suggest to proceed differently. We fix $\Theta_{0}=0$ and, for any $k \geq 0$, if $\Theta_{2 k+1}=\Lambda_{i}\left(\Theta_{2 k}\right)>\Theta_{2 k}$ (resp., $\Theta_{2 k+1}<\Theta_{2 k}$ ) we take some $\Theta_{2 k+2} \in\left(\Theta_{2 k}, \Theta_{2 k+1}\right)$ (resp., $\Theta_{2 k+2} \in$ $\left.\left(\Theta_{2 k+1}, \Theta_{2 k}\right)\right)$. With this procedure we constructed a new sequence such that $\left(\Theta_{2 k+1}-\Theta_{2 k}\right) \rightarrow 0$ as $k \rightarrow \infty$, that is,

$$
\exists \bar{\Theta}_{i}=\lim _{n \rightarrow \infty} \Theta_{n} \quad(i=0,1,2,3),
$$

where the index $i$ identifies which of the $\Upsilon_{i}$ 's is used to construct the sequence, see (7.5).

We numerically computed these limits for different values of $d$, see Table 9 , where we only report the first digits of $\bar{\Theta}_{i}$ : the results turned out to be very sensitive to modifications of these values up to 4 more digits and our numerical procedure stopped precisely when $\Theta_{2 k}$ and $\Theta_{2 k+1}$ had the first 7 nonzero digits coinciding.

TABLE 9. Approximate value of the optimal constants $\bar{\Theta}_{i}$ in (7.6), case of a single coach.

\begin{tabular}{|c|c|c|c|c|}
\hline$d$ & 0 & 50 & 100 & 225 \\
\hline $\bar{\Theta}_{0}$ & $1.131 \cdot 10^{-6}$ & $1.021 \cdot 10^{-5}$ & $1.74 \cdot 10^{-5}$ & $2.509 \cdot 10^{-5}$ \\
\hline $\bar{\Theta}_{1}$ & $9.842 \cdot 10^{-7}$ & $1.016 \cdot 10^{-5}$ & $1.729 \cdot 10^{-5}$ & $2.477 \cdot 10^{-5}$ \\
\hline $\bar{\Theta}_{2}$ & $9.843 \cdot 10^{-7}$ & $1.017 \cdot 10^{-5}$ & $1.73 \cdot 10^{-5}$ & $2.477 \cdot 10^{-5}$ \\
\hline $\bar{\Theta}_{3}$ & $9.672 \cdot 10^{-7}$ & $1.005 \cdot 10^{-5}$ & $1.723 \cdot 10^{-5}$ & $2.492 \cdot 10^{-5}$ \\
\hline
\end{tabular}

It appears that the best approximation of $\bar{\Theta}_{0}$ is $\bar{\Theta}_{2}$ if $d=0,50,100$ (asymmetric load), whereas it is $\bar{\Theta}_{3}$ if $d=225$ (almost symmetric load). The most frequently used approximation $\bar{\Theta}_{1}$ is never the best one.

The corresponding solutions of (7.3), which we denote by $v_{i}$, satisfy the linear equation

$$
v_{i}^{\prime \prime \prime \prime}(s)-\left(90.72+7078 \bar{\Theta}_{i}\right) v_{i}^{\prime \prime}(s)+650999 \bar{\Theta}_{i}=491 \psi_{d}(s) \quad \forall s \in(0,2)
$$

and can be explicitly computed by means of Proposition 5.5. Instead of giving the analytic form, we plot the differences between these solutions. Since $\bar{\Theta}_{1} \approx$ $\bar{\Theta}_{2}$ in all the above experiments, we also found that $v_{1} \approx v_{2}$. Therefore, in Figure 4 we only plot the functions $v_{2}-v_{0}$ and $v_{3}-v_{0}$.

We now take as live load a freight train of length $230 \mathrm{~m}$ having a weight density of $20 \mathrm{kN} / \mathrm{m}$, that is,

$$
p(x)=20 \chi_{(d, d+230)} \mathrm{kN} / \mathrm{m}, \quad 0<d<230,
$$

where $\chi_{(d, d+230)}$ is the characteristic function of the interval $(d, d+230)$. We consider both the cases where the train occupies the first half of the span 

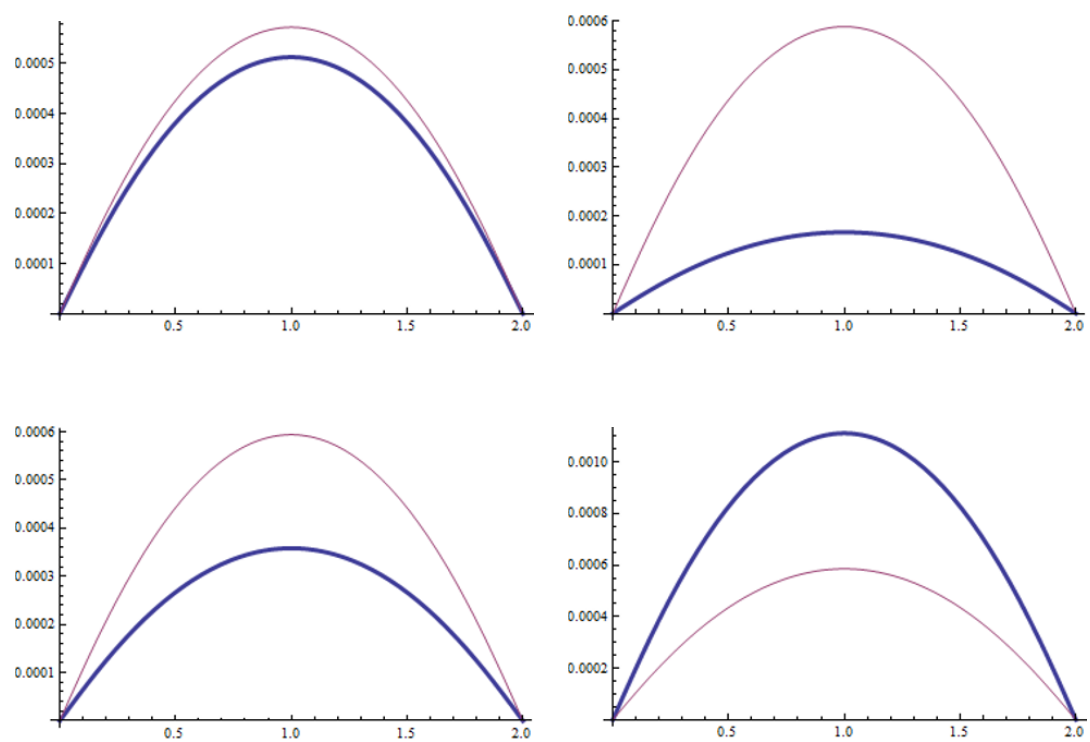

Figure 4. Plots of the functions $v_{2}-v_{0}$ (thick) and $v_{3}-v_{0}$ (thin) for $d=0,50,100,250$ (from left to right).

$(d=0)$ and the case where the train is in the middle of the span $(d=115)$. With the same scaling as above, instead of (7.3) we obtain

$v^{\prime \prime \prime \prime}(s)-\left(90.72+7078 \Upsilon_{i}(v)\right) v^{\prime \prime}(s)+650999 \Upsilon_{i}(v)=982 \psi_{\delta}(s) \quad \forall s \in(0,2)$,

where $\psi_{\delta}$ is the characteristic function of $(\delta, 1+\delta)$ with $\delta=0$ or $\delta=\frac{1}{2}$. We solve equation (7.7) by replacing $\Upsilon_{i}(v)$ with $\Theta$, that is, we consider again (7.4), where

$$
\alpha^{2}:=90.72+7078 \Theta, \quad f(s):=491 \psi_{\delta}(s)-650999 \Theta .
$$

By Proposition 5.5, this linear equation, complemented with hinged boundary conditions, admits a unique solution $V_{\Theta}$ given by

$$
\begin{aligned}
V_{\Theta}(s)= & \left(\frac{491(3-2 \delta)}{2}-650999 \Theta\right) \frac{s}{\alpha^{2}} \\
& +\frac{650999 \Theta}{2 \alpha^{2}} s^{2}+\frac{650999 \Theta}{\alpha^{4}}(1-\cosh (\alpha s)) \\
& +\left[650999 \Theta(\cosh (2 \alpha)-1)-1964 \sinh \frac{\alpha}{2} \sinh \frac{\alpha(3-2 \delta)}{2}\right] \\
& \times \frac{\sinh (\alpha s)}{\alpha^{4} \sinh (2 \alpha)}+982 \Psi_{\delta, \Theta}(s)
\end{aligned}
$$


where

$$
\Psi_{\delta, \Theta}(s)= \begin{cases}0 & \text { if } 0 \leq s \leq \delta, \\ \frac{1}{\alpha^{4}}(\cosh [\alpha(s-\delta)]-1)-\frac{(s-\delta)^{2}}{2 \alpha^{2}} & \text { if } \delta<s<\delta+1, \\ \frac{2}{\alpha^{4}} \sinh \frac{\alpha}{2} \sinh \frac{\alpha(2 s-2 \delta-1)}{2}+\frac{1+2 \delta-2 s}{2 \alpha^{2}} & \text { if } \delta+1 \leq s \leq 2 .\end{cases}
$$

We then define again $\Lambda_{i}$ as in (7.5) and we find out that it has an unstable fixed point, that is, the behavior of the sequence $\Theta_{n}$ is well described by Figure 3. With the same algorithm previously described, we are again able to construct a converging sequence and we denote again by $\bar{\Theta}_{i}$ its limit, see (7.6), where the index $i$ identifies which of the $\Upsilon_{i}$ 's is used to construct the sequence, see (7.5). We numerically computed these limits for $d=0$ (train in the first half of the span) and $d=115$ (train in the middle of the span), see Table 10, where we only report the first digits of $\bar{\Theta}_{i}$ : again, the results turned out to be very sensitive to modifications of these values up to 4 more digits and our numerical procedure stopped when $\Theta_{2 k}$ and $\Theta_{2 k+1}$ had the first 7 nonzero digits coinciding.

TABLE 10. Approximate value of the optimal constants $\bar{\Theta}_{i}$ in (7.6), case of a whole train.

\begin{tabular}{|c|c|c|}
\hline$d$ & 0 & 115 \\
\hline $\bar{\Theta}_{0}$ & $7.582 \cdot 10^{-4}$ & $1.047 \cdot 10^{-3}$ \\
\hline $\bar{\Theta}_{1}$ & $7.538 \cdot 10^{-4}$ & $1.042 \cdot 10^{-3}$ \\
\hline $\bar{\Theta}_{2}$ & $7.582 \cdot 10^{-4}$ & $1.044 \cdot 10^{-3}$ \\
\hline $\bar{\Theta}_{3}$ & $7.538 \cdot 10^{-4}$ & $1.046 \cdot 10^{-3}$ \\
\hline
\end{tabular}

Again, the best approximation of $\bar{\Theta}_{0}$ is $\bar{\Theta}_{2}$ if $d=0$ (asymmetric load), whereas it is $\bar{\Theta}_{3}$ if $d=115$ (symmetric load). And, again, $\bar{\Theta}_{1}$ is never the best one.

The corresponding solutions of (7.7), which we denote by $v_{i}$, satisfy the linear equation

$$
v_{i}^{\prime \prime \prime \prime}(s)-\left(90.72+7078 \bar{\Theta}_{i}\right) v_{i}^{\prime \prime}(s)+650999 \bar{\Theta}_{i}=982 \psi_{\delta}(s) \quad \forall s \in(0,2)
$$

and can be explicitly computed by means of Proposition 5.5. In Figure 5 we plot the differences between these solutions. When $d=0$ we have $\bar{\Theta}_{1} \approx \bar{\Theta}_{3}$ and $\bar{\Theta}_{2} \approx \bar{\Theta}_{0}$ : whence, we only plot the function $v_{1}-v_{0}$ since $v_{3}-v_{0}$ is almost identical and $v_{2}-v_{0}$ is almost 0 . When $d=115$ we plot the three differences $v_{i}-v_{0}(i=1,2,3)$ so that it appears clearly how they are ordered.

By scaling, similar pictures can be obtained for the original solutions $w_{i}$ of (7.1) after undoing the change of variables (7.2). 

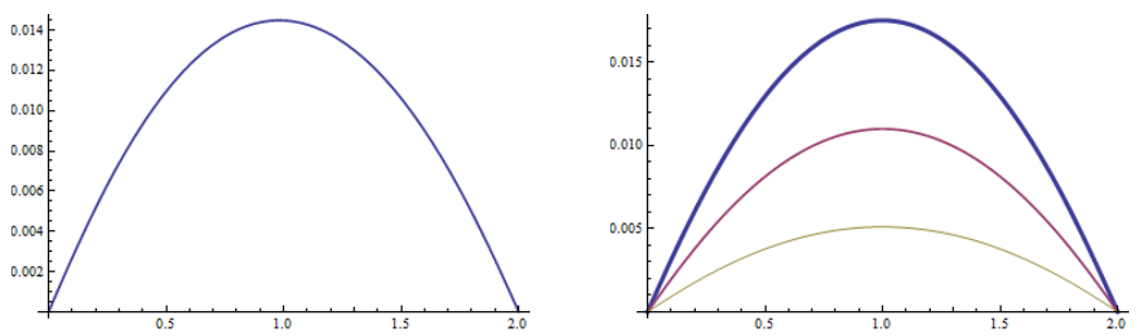

Figure 5. On the left, plot of the function $v_{1}-v_{0}$ for $d=0$. On the right, plots of the functions $v_{1}-v_{0}$ (thick), $v_{2}-v_{0}$ (intermediate), $v_{3}-v_{0}$ (thin) for $d=115$.

\section{Proof of Theorem 5.1}

We first prove the inequality

$$
\|u\|_{\infty} \leq\left(\frac{L}{\pi}\right)^{3 / 2}\left\|u^{\prime \prime}\right\|_{2} \quad \forall u \in H^{2}(0, L) \cap H_{0}^{1}(0, L) .
$$

The main ingredient to obtain (8.1) is a special version of the GagliardoNirenberg inequality $[9,19]$; since we are interested in the value of the estimating constant and since we were unable to find one in literature, we give its proof. We do not know if the constant is optimal. We first claim that

$$
\|u\|_{\infty}^{2} \leq\|u\|_{2}\left\|u^{\prime}\right\|_{2} \quad \forall u \in H_{0}^{1}(0, L) .
$$

Since symmetrization leaves $L^{p}$-norms of functions invariant and decreases the $L^{p}$-norms of the derivatives, see e.g. [1, Theorem 2.7], for the proof of (8.2) we may restrict our attention to functions which are symmetric, positive and decreasing with respect to the center of the interval. If $u$ is one such function, we have

$$
\begin{aligned}
\int_{0}^{L / 2} u(\tau) u^{\prime}(\tau) d \tau & =\int_{0}^{L / 2}\left|u(\tau) u^{\prime}(\tau)\right| d \tau=\int_{L / 2}^{L}\left|u(\tau) u^{\prime}(\tau)\right| d \tau \\
& =\frac{1}{2} \int_{0}^{L}\left|u(\tau) u^{\prime}(\tau)\right| d \tau .
\end{aligned}
$$

Therefore, we have

$$
\begin{aligned}
\|u\|_{\infty}^{2} & =u\left(\frac{L}{2}\right)^{2}=\int_{0}^{L / 2}\left[u(\tau)^{2}\right]^{\prime} d \tau=2 \int_{0}^{L / 2} u(\tau) u^{\prime}(\tau) d \tau \\
& =\int_{0}^{L}\left|u(\tau) u^{\prime}(\tau)\right| d \tau \leq\|u\|_{2}\left\|u^{\prime}\right\|_{2},
\end{aligned}
$$

where we used Hölder's inequality. This proves (8.2).

Then we recall two Poincaré-type inequalities:

$$
\|u\|_{2} \leq \frac{L^{2}}{\pi^{2}}\left\|u^{\prime \prime}\right\|_{2}, \quad\left\|u^{\prime}\right\|_{2} \leq \frac{L}{\pi}\left\|u^{\prime \prime}\right\|_{2} \quad \forall u \in H^{2}(0, L) \cap H_{0}^{1}(0, L) .
$$


The proof of (8.1) follows by combining these inequalities with (8.2).

Next, we multiply (5.13) by $u(x)$ and integrate by parts to obtain

$$
\left\|u^{\prime \prime}\right\|_{2}^{2}+\alpha^{2}\left\|u^{\prime}\right\|_{2}^{2}=\int_{0}^{L} f(x) u(x) d x \leq\|f\|_{1}\|u\|_{\infty},
$$

where we used Hölder's inequality. Neglecting the positive term $\alpha^{2}\left\|u^{\prime}\right\|_{2}^{2}$ and using (8.1), we get

$$
\frac{\pi^{3}}{L^{3}}\|u\|_{\infty}^{2} \leq\left\|u^{\prime \prime}\right\|_{2}^{2} \leq\|f\|_{1}\|u\|_{\infty}
$$

which readily gives the following $L^{\infty}$-bound for the solution of (5.13):

$$
\|u\|_{\infty} \leq \frac{L^{3}}{\pi^{3}}\|f\|_{1}
$$

Next, we consider the closed (convex) ball

$$
B:=\left\{v \in C^{0}[0, L] ;\|v\|_{\infty} \leq d\|p\|_{1}\right\},
$$

where

$$
d:=\frac{L^{3}}{\pi^{3}-b c L^{5}}>0
$$

and the positivity of $d$ is a consequence of (5.3). We define an operator $T$ : $B \rightarrow C^{0}[0, T]$ as follows. For any $v \in B$ we denote by $w=T v$ the unique solution $w \in W^{4,1}(0, L) \cap H_{0}^{1}(0, L)$ of the problem

$$
\begin{gathered}
w^{\prime \prime \prime \prime}(x)-(a+h(v)) w^{\prime \prime}(x)+b h(v)=p(x) \text { for } x \in(0, L), \\
w(0)=w(L)=w^{\prime \prime}(0)=w^{\prime \prime}(L)=0 .
\end{gathered}
$$

Note that if $v \in B$, then

$$
\alpha^{2}:=a+h(v) \geq a-c\|v\|_{1}>a-c L\|v\|_{\infty} \geq a-c d L\|p\|_{1} \geq 0,
$$

where we used (5.2) (first inequality), Hölder's inequality (second), $v \in B$ (third), (5.4) (fourth). Putting

$$
f(x):=p(x)-b h(v)
$$

so that $f \in L^{1}(0, L)$, Proposition 5.5 then ensures that there exists a unique solution $w \in W^{4,1}(0, L) \cap H_{0}^{1}(0, L)$ of (8.5). Together with the compact embedding $W^{4,1}(0, L) \Subset C^{0}[0, L]$, this shows that

$$
\text { the map } T: B \rightarrow C^{0}[0, L] \text { is well defined and compact. }
$$

Moreover, by (8.4) we know that

$$
\begin{aligned}
\|w\|_{\infty} & \leq \frac{L^{3}}{\pi^{3}}\|p-b h(v)\|_{1} \leq \frac{L^{3}}{\pi^{3}}\left(\|p\|_{1}+b L|h(v)|\right) \\
& \leq \frac{L^{3}}{\pi^{3}}\left(\|p\|_{1}+b c L\|v\|_{1}\right) \\
& \leq \frac{L^{3}}{\pi^{3}}\left(\|p\|_{1}+b c L^{2}\|v\|_{\infty}\right) \\
& \leq \frac{L^{3}}{\pi^{3}}\left(1+b c d L^{2}\right)\|p\|_{1}=d\|p\|_{1}
\end{aligned}
$$

(by Hölder's inequality)

(since $v \in B$ ). 
This shows that, in fact, $T(B) \subset B$. Combined with (8.6) and with the Schauder fixed point theorem (see, e.g., [13, Section 6, Theorem 3.2]), this proves that the map $T$ admits a fixed point in $B$ which is a solution of (5.1).

\section{Proof of Theorem 5.2}

Take $u \in H^{2}(0, L) \cap H_{0}^{1}(0, L)$; since $u(0)=u(L)=0$ and $u \in C^{1}[0, L]$, by the Fermat theorem we know that there exists $x_{0} \in(0, L)$ such that $u^{\prime}\left(x_{0}\right)=0$. Therefore,

$$
\left|u^{\prime}(x)\right|=\left|\int_{x_{0}}^{x} u^{\prime \prime}(t) d t\right| \leq \int_{0}^{L}\left|u^{\prime \prime}(t)\right| d t \leq \sqrt{L}\left\|u^{\prime \prime}\right\|_{2} \quad \forall x \in(0, L)
$$

which, by arbitrariness of $x$, proves that

$$
\left\|u^{\prime}\right\|_{\infty} \leq \sqrt{L}\left\|u^{\prime \prime}\right\|_{2} \quad \forall u \in H^{2}(0, L) \cap H_{0}^{1}(0, L) .
$$

Similarly, we find that $|u(x)| \leq \int_{0}^{L}\left|u^{\prime}(t)\right| d t$ and therefore

$$
\|u\|_{\infty} \leq L\left\|u^{\prime}\right\|_{\infty} \quad \forall u \in H^{2}(0, L) \cap H_{0}^{1}(0, L) .
$$

If we multiply (5.13) by $u(x)$ and integrate by parts, we obtain

$$
\left\|u^{\prime \prime}\right\|_{2}^{2}<\left\|u^{\prime \prime}\right\|_{2}^{2}+\alpha^{2}\left\|u^{\prime}\right\|_{2}^{2} \leq\|f\|_{1}\|u\|_{\infty} \leq L\|f\|_{1}\left\|u^{\prime}\right\|_{\infty},
$$

where we used (9.2). Using (9.1), we get the following bound for the derivative of the solution of (5.13):

$$
\left\|u^{\prime}\right\|_{\infty} \leq L^{2}\|f\|_{1} .
$$

Let $C_{0}^{1}[0, L]=\left\{v \in C^{1}[0, L] ; v(0)=v(L)=0\right\}$ and consider the closed (convex) ball

$$
B:=\left\{v \in C_{0}^{1}[0, L] ;\left\|v^{\prime}\right\|_{\infty} \leq d\|p\|_{1}\right\},
$$

where

$$
d:=\frac{L^{2}}{1-b c L^{4}}>0,
$$

and the positivity of $d$ is a consequence of (5.6). We define an operator $T$ : $B \rightarrow C_{0}^{1}[0, T]$ as follows. For any $v \in B$ we denote by $w=T v$ the unique solution $w \in W^{4,1}(0, L) \cap C_{0}^{1}[0, L]$ of problem (8.5). Note that if $v \in B$, then

$$
\alpha^{2}:=a+h(v) \geq a-c\left\|v^{\prime}\right\|_{1}>a-c L\left\|v^{\prime}\right\|_{\infty} \geq a-c d L\|p\|_{1} \geq 0,
$$

where we used (5.5) (first inequality), Hölder's inequality (second), $v \in B$ (third), (5.7) (fourth). Putting

$$
f(x):=p(x)-b h(v)
$$

so that $f \in L^{1}(0, L)$, Proposition 5.5 then ensures that there exists a unique solution $w \in W^{4,1}(0, L) \cap C_{0}^{1}[0, L]$ of (8.5). Together with the compact embedding $W^{4,1}(0, L) \Subset C^{1}[0, L]$, this shows that

$$
\text { the map } T: B \rightarrow C_{0}^{1}[0, L] \text { is well defined and compact. }
$$


By (9.3) we know that

$$
\begin{aligned}
\left\|w^{\prime}\right\|_{\infty} & \leq L^{2}\|p-b h(v)\|_{1} \leq L^{2}\left(\|p\|_{1}+b L|h(v)|\right) \\
& \leq L^{2}\left(\|p\|_{1}+b c L\left\|v^{\prime}\right\|_{1}\right) \\
& \leq L^{2}\left(\|p\|_{1}+b c L^{2}\left\|v^{\prime}\right\|_{\infty}\right) \\
& \leq L^{2}\left(1+b c d L^{2}\right)\|p\|_{1}=d\|p\|_{1}
\end{aligned}
$$

(by Hölder's inequality)

(since $v \in B$ ).

This shows that, in fact, $T(B) \subset B$. Combined with (9.4) and with the Schauder fixed point theorem (see, e.g., [13, Section 6, Theorem 3.2]), this proves that the map $T$ admits a fixed point in $B$ which is a solution of (5.1).

\section{Proof of Theorem 5.4}

Consider the closed ball

$$
B:=\left\{v \in H^{2}(0, L) \cap H_{0}^{1}(0, L) ;\left\|v^{\prime \prime}\right\|_{2} \leq d\|p\|_{1}\right\}
$$

where

$$
d:=\frac{\pi L^{5 / 2}}{\pi^{5 / 2}-b c L^{5 / 2}}>0,
$$

and the positivity of $d$ is a consequence of (5.9). We define an operator $T$ : $B \rightarrow H^{2}(0, L) \cap H_{0}^{1}(0, L)$ as follows. For any $v \in B$ we denote by $w=T v$ the unique solution $w \in W^{4,1}(0, L) \cap H_{0}^{1}(0, L)$ of $(8.5)$.

Note that

$$
\alpha^{2}:=a+h(v) \geq a-c\left\|v^{\prime \prime}\right\|_{2} \geq a-c d\|p\|_{1}>0 \quad \forall v \in B,
$$

where we used (5.8) (first inequality), $v \in B$ (second), (5.10) (third). Putting

$$
f(x):=p(x)-b h(v)
$$

so that $f \in L^{1}(0, L)$, Proposition 5.5 then ensures that there exists a unique solution $w \in W^{4,1}(0, L) \cap H_{0}^{1}(0, L)$ of $(8.5)$. Together with the compact embedding $W^{4,1}(0, L) \Subset H^{2}(0, L)$, this shows that

the map $T: B \rightarrow H^{2}(0, L) \cap H_{0}^{1}(0, L)$ is well defined and compact.

Let $v_{1}, v_{2} \in B$ and let $w_{i}=T v_{i}$ for $i=1,2$. Then $w_{i}$ satisfies

$$
\begin{gathered}
w_{i}^{\prime \prime \prime \prime}(x)-\left(a+h\left(v_{i}\right)\right) w_{i}^{\prime \prime}(x)+b h\left(v_{i}\right)=p(x) \quad \text { for } x \in(0, L), \\
w_{i}(0)=w_{i}(L)=w_{i}^{\prime \prime}(0)=w_{i}^{\prime \prime}(L)=0 .
\end{gathered}
$$

Multiplying (10.3) by $w_{i}$ and integrating by parts, we obtain the following estimate:

$$
\left\|w_{i}^{\prime \prime}\right\|_{2}^{2} \leq\left(b L\left|h\left(v_{i}\right)\right|+\|p\|_{1}\right)\left\|w_{i}\right\|_{\infty} \leq\left(\frac{L}{\pi}\right)^{3 / 2}\left(b c L\left\|v_{i}^{\prime \prime}\right\|_{2}+\|p\|_{1}\right)\left\|w_{i}^{\prime \prime}\right\|_{2},
$$

where we used (10.1) and Hölder's inequality (first inequality), (5.8) and (8.1) (second). Whence, since $v_{i} \in B$, we finally obtain

$$
\left\|w_{i}^{\prime \prime}\right\|_{2} \leq\left(\frac{L}{\pi}\right)^{3 / 2}(b c d L+1)\|p\|_{1}
$$


Put $v:=v_{1}-v_{2}$ and $w:=w_{1}-w_{2}$. Then, subtracting the two equations in $(10.3)$, we find

$$
w^{\prime \prime \prime \prime}(x)-\left(a+h\left(v_{1}\right)\right) w^{\prime \prime}(x)=\left[h\left(v_{1}\right)-h\left(v_{2}\right)\right]\left(-b+w_{2}^{\prime \prime}(x)\right) \text { for } x \in(0, L) .
$$

Let us multiply this equation by $w$ and integrate by parts to obtain

$$
\left\|w^{\prime \prime}\right\|_{2}^{2} \leq\left[h\left(v_{1}\right)-h\left(v_{2}\right)\right] \int_{0}^{L}\left(-b+w_{2}^{\prime \prime}(x)\right) w^{\prime \prime}(x) d x
$$

where we dropped the term $\alpha^{2}\left\|w^{\prime}\right\|_{2}^{2}$ in view of (10.1). By (5.8) and Hölder's inequality (twice) we get

$$
\left\|w^{\prime \prime}\right\|_{2}^{2} \leq c\left\|v_{1}^{\prime \prime}-v_{2}^{\prime \prime}\right\|_{2}\left(b\left\|w^{\prime \prime}\right\|_{1}+\left\|w_{2}^{\prime \prime}\right\|_{2}\left\|w^{\prime \prime}\right\|_{2}\right) \leq c\left\|v^{\prime \prime}\right\|_{2}\left(b \sqrt{L}+\left\|w_{2}^{\prime \prime}\right\|_{2}\right)\left\|w^{\prime \prime}\right\|_{2} \text {. }
$$

Whence, by (10.4)

$$
\left\|w^{\prime \prime}\right\|_{2} \leq c\left\|v^{\prime \prime}\right\|_{2}\left[b \sqrt{L}+\left(\frac{L}{\pi}\right)^{3 / 2}(b c d L+1)\|p\|_{1}\right]=(1-\varepsilon)\left\|v^{\prime \prime}\right\|_{2},
$$

where

$\varepsilon:=c(1+b c d L)\left(\frac{L}{\pi}\right)^{3 / 2}\left[\left(\frac{\pi}{L}\right)^{3 / 2} \frac{\left(\pi^{5 / 2}-b c L^{5 / 2}\right)(1-b c \sqrt{L})}{c\left(\pi^{5 / 2}-b c L^{5 / 2}+b c \pi L^{7 / 2}\right)}-\|p\|_{1}\right]>0$

in view of (5.10). This shows that $T(B) \subset B$ is a contractive map. Whence by the Banach contraction principle (see, e.g., [13, Section 1, Theorem 1.1]) it admits a unique fixed point in $B$ which is a solution of (5.1).

\section{Conclusions and open problems}

In spite of the double inequality in (3.11), the explicit computations performed in Section 4 do not allow to infer a precise rule on which form of $h(w)$ better approximates the additional tension of cables in suspension bridges. We found both large and tiny percentage errors, both by excess and by defect, of the value $\Gamma(w)$. For these reasons, the approximations do not appear completely reliable. In our computations none between the three approximations $\Gamma_{i}$ seemed better than the others: an important result would then be to understand in which situation an approximation $\Gamma_{i}$ is better than the others.

The existence results in Section 5 are obtained by fixed point techniques. There are several alternative statements, depending on the explicit assumptions on $h$. Theorem 5.4 is perhaps the strongest result: not only it makes general assumptions on $h$, see (5.8), but also it gives a uniqueness statement for small solutions. The Counterexample 1 shows that Theorem 5.4 cannot be improved, the problem is ill-posed and further large solutions may exist. This gives rise to several natural questions. Under which assumptions on $h$ can one ensure existence and uniqueness of solutions of (1.1)? In this situation, can the solution be approximated by a suitable constructive sequence? 
Concerning the last question, we suggested in Section 6 that a sequence of approximate solutions $\left\{w_{n}\right\}$ might be tested with the numerical sequence $\left\{h\left(w_{n}\right)\right\}$. We numerically found that, for suitable values of the parameters, this sequence admits a unique stable fixed point qualitatively described by Figure 2. However, when the parameters are in the range of actual bridges, in Section 7 we found that the fixed point is unstable, see Figure 3, and an iterative procedure seems not possible. We therefore suggested a different algorithm which allowed to find a fixed point. Our numerical results also suggest several questions. Under which assumptions on the parameters is the iterative scheme convergent? Are there better algorithms able to manage both the stable and unstable cases? Can these algorithms detect multiple fixed points?

On the whole, we believe that some further research is needed in order to formulate a sound and complete existence and uniqueness theory for the Melan equation (1.1) and to determine stable approximation algorithms.

\section{Acknowledgement}

This project was supported by King Saud University, Deanship of Scientific Research, College of Science Research Center.

\section{References}

[1] F. Almgren, Jr., J. Frederick and E. Lieb, Symmetric decreasing rearrangement is sometimes continuous. J. Amer. Math. Soc. 2 (1989), 683-773.

[2] O. H. Ammann, T. von Kármán and G. B. Woodruff, The Failure of the Tacoma Narrows Bridge. Federal Works Agency, Washington DC, 1941.

[3] J. M. W. Brownjohn, Observations on non-linear dynamic characteristics of suspension bridges. Earthquake Engineering \& Structural Dynamics 23 (1994), 1351-1367.

[4] C. A. Castigliano, Nuova teoria intorno all'equilibrio dei sistemi elastici. Atti Acc. Sci. Torino, Cl. Sci. Fis. Mat. Nat. 11 (1875-76), 127-286.

[5] C. A. Castigliano, Théorie de l'équilibre des systèmes élastiques et ses applications. A. F. Negro, Torino, 1879.

[6] M. Como, Stabilità aerodinamica dei ponti di grande luce. Introduzione all'ingegneria strutturale. E. Giangreco, UTET, Torino, 2002.

[7] M. Como, S. Del Ferraro and A. Grimaldi, A parametric analysis of the flutter instability for long span suspension bridges. Wind and Structures 8 (2005), $1-12$.

[8] Q. A. Dang and V. T. Luan, Iterative method for solving a nonlinear fourth order boundary value problem. Comput. Math. Appl. 60 (2010), 112-121.

[9] E. Gagliardo, Proprietà di alcune classi di funzioni in più variabili. Ricerche Mat. 7 (1958), 102-137. 
[10] F. Gazzola, Nonlinearity in oscillating bridges. Electron. J. Differential Equations 211 (2013), 1-47.

[11] F. Gazzola, H.-Ch. Grunau and G. Sweers, Polyharmonic Boundary Value Problems. Lecture Notes in Math. 1991, Springer, Berlin, 2010.

[12] F. Gazzola and R. Pavani, Wide oscillations finite time blow up for solutions to nonlinear fourth order differential equations. Arch. Ration. Mech. Anal. 207 (2013), 717-752.

[13] A. Granas and J. Dugundji, Fixed Point Theory. Springer Monogr. Math., Springer, New York, 2003.

[14] T. von Kármán and M. A. Biot, Mathematical Methods in Engineering: An Introduction to the Mathematical Treatment of Engineering Problems. McGrawHill, New York, 1940.

[15] W. Lacarbonara, Nonlinear Structural Mechanics. Springer, New York, 2013.

[16] J. E. Luco and J. Turmo, Effect of hanger flexibility on dynamic response of suspension bridges. J. Eng. Mech. 136 (2010), 1444-1459.

[17] J. Melan, Theory of arches and suspension bridges. Myron Clark Publ. Comp., London, 1913 (German original third edition: Handbuch der Ingenieurwissenschaften 2, 1906).

[18] C. L. Navier, Mémoire sur les ponts suspendus. Imprimerie Royale, Paris, 1823.

[19] L. Nirenberg, On elliptic partial differential equations. Ann. Scuola Norm. Sup. Pisa 13 (1959), 115-162.

[20] M. R. Ohm, H. Y. Lee and J. Y. Shin, Error estimates of finite-element approximations for a fourth-order differential equation. Comput. Math. Appl. 52 (2006), 283-288.

[21] R. H. Plaut and F. M. Davis, Sudden lateral asymmetry and torsional oscillations of section models of suspension bridges. J. Sound and Vibration 307 (2007), 894-905.

[22] W. Podolny, Cable-suspended bridges. In: Structural Steel Designers Handbook: AISC, AASHTO, AISI, ASTM, AREMA, and ASCE-07 Design Standards, 5th ed., McGraw-Hill, New York, 2011.

[23] W. J. M. Rankine, A Manual of Applied Mechanics. Charles Griffin \& Company, London, 1858.

[24] B. Semper, A mathematical model for suspension bridge vibration. Math. Comput. Modelling 18 (1993), 17-28.

[25] B. Semper, Finite element approximation of a fourth order integro-differential equation. Appl. Math. Lett. 7 (1994), 59-62.

[26] D. B. Steinman, A Practical Treatise on Suspension Bridges: Their Design, Construction and Erection. John Wiley \& Sons, New York, 1922.

[27] S. P. Timoshenko, Theory of suspension bridges. I. J. Franklin Inst. 235 (1943), 213-238.

[28] S. P. Timoshenko, Theory of suspension bridges. II. J. Franklin Inst. 235 (1943), $327-349$. 
[29] S. P. Timoshenko and D. H. Young, Theory of Structures. McGraw-Hill Kogakusha, Tokyo, 1965.

[30] G. P. Wollmann, Preliminary analysis of suspension bridges. J. Bridge Eng. 6 (2001), 227-233. 